\title{
Malaria Elimination in China: Innovative Three-Layer Strategy Applied to the Outbreak of Indigenous Cases in Sanya, Hainan
}

Yuchun Li

Hainan provincial center for disease control and prevention https://orcid.org/0000-0001-6951-4403

Yingjuan Huang

Tangshan City Center for Disease Control and Prevention

Renqiang Chen

Sanya city for disease control and prevention

Weizhen Huang

Wuzhishan city for disease control and prevention

Huanzhi Xu

Wuzhishan city for disease control and prevention

Rongshen Ye

Baoting county center for disease control and prevention

Shaoling Huang

wanning center for disease control and prevention

Ji Zhen

dongfang city for disease control and prevention

Xiaodan Wen

danzhou city for disease contol and prevention

Guoyi Wang

Qiongzhong county for disease control and prevention

Yong Liu

tunchang county for disease control and prevention

Haishan Li

ledong county for disease control and prevention

Zaichun Zhen

changjiang county for diease control and prevention

Jian Wang

Baisha county for disease control and prevention

Guoshen Wang

qionghai county for disease control and prevention

Chong Chen

lingshui institute of health supervision

Wen Zeng

hainan center for disease control and prevention

Feng Meng

hainan provicial center for disease control and prevention

Xiaoming Huang

hainan rovincial center for disease control and prevention

Guangze Wang

Hainan Provincial Center for Disease Control and Prevention

Bing Yang

Hainan Provincial Center for Disease Control and Prevention

Yan Chen ( $\nabla$ jkban702@163.com )

hainan Provincial Center for Disease Control and Prevention

\section{Research Article}

Keywords: Three-Layer Strategy, Outbreak, malaria, elimination

Posted Date: January 21st, 2022

DOI: https://doi.org/10.21203/rs.3.rs-1242449/v1 


\section{Abstract}

\section{Background}

Forest-goers have been the most severely infected by malaria in Hainan since the 1990s. In 2015, there were successively 6 cases of $P$. malariae infection as an outbreak by forest-goers in Sanya city, Hainan Province. According to the 1-3-7 approach, active case detection(ACD) was performed with guidance from information from Case 1. Cases 2-4 had the same epidemiological history as Case 1 and were similarly confirmed to be infected with $P$. malariae. However, the clues from Case 1 came to an end and it was uncertain whether there were other potential infection sources or whether transmission was established in local villages.

\section{Methods}

There was no detailed strategy for the outbreak until 2015, although the 1-3-7 approach was certified to be effective for sporadic case. A retrospective study was conducted on this epidemic of $P$. malariae transmission among forest-goers in Hainan, 2015. A three-layer strategy (TLS) that integrated malaria joint prevention and control strategies(JPCS) and the 1-3-7 approach was first generated and applied to the disposal of the outbreak in Sanya in 2015, and its was also applied to strengthen epidemic measures to prevent emergence by residual foci from 2016 to 2018 . The data were entered by Microsoft Office Excel 2007 for collection and statistic analysis by SPSS.

\section{Results}

With guideline of TLS, three villages in Gaofeng town, Sanya city and Seven villages in Xinzheng Town, Baoting County, were selected as the first layer with design of TLS. In the first layer, 1,823 blood slides and PCR screening from villages in Baoting and Sanya were conducted on all residents by active case detection (ACD) and four cases were identified. In the second layers, passive case detection (PCD) and ACD for forest-goers were conducted and 7,831 blood slides were screened on all forest-goers. In the third layer, PCD was conducted for forest-goers, and 95,350 blood slides were screened on all forest-goers. Meanwhile, vector surveillance and control were also conducted to support foci disposals and to minimize the possibility of transmission. From 2016 to 2018 , TLS was also applied to strengthen epidemic measures to prevent emergence by residual foci. In the first layer, chemoprophylaxis and ACD were conducted on residents in the villages of the foci and the surrounding villages, and $89.5 \%$ residents accepted chemoprophylaxis. Chemoprophylaxis and ACD were conducted and $79.1 \%$ received chemoprophylaxis while seeking forest-goers in the second layer. In the third layer, $89.6 \%$ received chemoprophylaxis while seeking forest-goers.

\section{Conclusions}

The innovative strategy of TLS not only was successfully applied in an epidemic response strategy in Sanya in 2015 but also was implemented in a strengthened intervention on forest-goers from 2016 to 2018 and finally achieved the goal of eliminating malaria in 2020 as scheduled. TLS is suitable for outbreak by indigenous case of unknown causes or for sporadic cases with defects in epidemic information, while 1-3-7 approach is suitable for sporadic cases which have clearly epidemic clues. Furthermore, TLS could be one of the major achievements after 1-3-7 approach, targeting outbreak in elimination malaria.

\section{Background}

Historically, malaria has been one of the most important infectious diseases in P.R.China. Hainan and Yunnan provinces were the main malaria transmission areas in P.R. China. Prior to 2010, indigenous cases of $P$. falciparum and $P$. vivax were still frequently detected in Hainan. Hainan is characterized geographically by mountains, hills, plateaus and plains. The tropical monsoon and marine climates jointly produce a generally warm temperature, within a suitable range not only for cultivating tropical plants (coconuts, areca nut and rubber trees, and more) and but also for the breeding of $A n$. dirus and $A n$. minimus[1]. Malaria cases in Hainan Province were mainly distributed in patches in the southwestern region of the island and a high-risk group for malaria infection can not be ignored in Hainan.

Forest-goers who sleep at night in the mountains include local residents and migrants are for picking and planting because of production and living. Forestgoers belong to a high-risk group of people who can become infected with malaria in Hainan[2]. Forest-goers have composed the main group of indigenous malaria infections in Hainan Province since 1990s. The infection rate was 30.9\% (95/307), which is more than twice that of the non-forest-goer residents (15.2\%) [3]. Malaria infection in forest-goers has been found to be related to the frequency of staying in the mountains and whether medicine was taken to prevent malaria, in addition the acceptance of the history of malaria control propaganda and the use of mosquito control measures [4-6]. Since the 1990s, Hainan Province has been engaged in malaria control and elimination for forest-goers for a long time. The strategy for the prevention and control of malaria, which had been supported by the Global Fund for ten years since 2003 , involved focusing on treatment in patients, timely tracking the disposal of foci or outbreaks, and applying insecticide-treated nets (ITNs) or distributing long-lasting insecticidal nets (LLINs) to reduce the incidence of malaria in forest-goers [7]. In 2010, Hainan joined the National Malaria Elimination Programme (NMEP) and involved eight Class I counties (endemic counties of $P$. falciparum) and ten Class II counties (endemic counties of P. vivax) in Hainan Province[8]. Since 2011, malaria elimination has been officially implemented in Hainan Province. The last indigenous malaria case of $P$. vivax was reported and the "1-3-7"approach (case reporting within 1 day, case investigation within 3 days, and focus investigation and action within 7 days) launched in 2012.

Since 2013, there have been only imported malaria cases in Hainan Province, and every sporadic foci has been classified and disposed under the guidelines of the 1-3-7 approach. However, indigenous cases of $P$. malariae appeared in 2015 [9]. According to the 1-3-7 approach, active case detection (ACD) was performed with guidance from information from Case 1. Three co-workers had the same epidemiological history as Case 1, but whether other potential infection sources or transmission was established in local villages was not known. There was no detailed strategy for the outbreak until 2015, although the 1- 
3-7 approach was certified as effective for imported malaria cases [10]. In response to the epidemic in the elimination stage, an innovative three-layer strategy (TLS) that integrated malaria joint prevention and control strategies(JPCS) and the 1-3-7 approach was first generated and applied to the disposal of the epidemic as an outbreak in 2015. Subsequently,TLS applied to the prevention of the emergence by residual foci from 2016 to 2018 . Finally, Hainan Province achieved the goal of eliminating malaria in 2019 and a WHO field certification of malaria elimination in 2021 [11]. This retrospective study on $P$. malariae transmission in 2015 as an example to illustrate and summarize the lessons learned about TLS processes from generation to application.

\section{Methods Study site}

Sanya city is located at the southern tip of Hainan Island, and it is in the tropical climate zone of the surrounding Pacific Ocean, with an annual average temperature of $25.7^{\circ} \mathrm{C}$. The tropical geographical climate environment is suitable for the reproduction of vectors of malaria throughout the year. Anopheles sinensis is considered to be a major vector in Sanya, but An. minimusis is also sometimes captured in mountainous regions. Sanya was the main endemic city and county of malaria in Hainan Province, with the recorded malarial parasites included $P$. vivax and $P$. falciparum[12]. Gaofeng township is located at the edge of Sanya city and borders Baoting, Ledong County and Wuzhishan City.

\section{Design of the TLS}

Due to the end of the clues from Case 1 and the dilemma of uncertain potential infection sources, a comprehensive strategy was essential for outbreak management rather than the disposal of each foci under the guidelines of the 1-3-7 approach. Case screening in these villages was the most effective method to solve this dilemma. Based on the geographical location of malaria cases, population characteristics, history of malaria joint defence and work requirements of the 1-3-7 approach, a three-layer strategy (TLS) that integrated malaria JPCS and the 1-3-7 approach was first generated for screening. Villages with cases and adjacent villages were regarded as the first layer in the prevention and control strategy. Gaofeng town and adjacent towns with cases were regarded as the second layer. Sanya city and adjacent counties or cities with cases were regarded as the third layer.

\section{TLS in disposal of the outbreak}

TLS firstly applied to the disposal of the outbreak. Briefly, the detailed disposal strategies are as follows: In the first layer, active case detection (ACD) was conducted mainly in the villages of the foci and the surrounding villages. In the second layer, the population of forest-goers was the main target, with an emphasis on the strategy of tracing clues, conducting spot visits to forest-goers in targeted townships, and conducting malaria blood slide screening for forest-goers who had a history of overnights in mountains with fever cases. In the third layer, the population of forest-goers was the main target, to provide them with health education and to alert them to the risk and to recommend to them not to overnight in the mountains at that time, while simultaneously reminding doctors to pay attention to forest-goers. To improve the accuracy, PCR detection was conducted on filtered blood collected from all samples in the first layer or suspicious samples in others layers. The partial gene of the SSU rRNA gene was amplified by PCR with Plasmodium-specific primers (rPLU1 and rPLU5) in Nest1 PCR and genus-specific primers (rPLU3 and rPLU4) in Nest2 PCR [14].

\section{TLS in strengthening of epidemic measures}

TLS was also applied to strengthen epidemic measures to prevent emergence by residual foci from 2016 to 2018 , to achieve the goal of eliminating malaria in 2020. Briefly, the detailed strengthen epidemic strategies are as follows: In the first layer, chemoprophylaxis and ACD were conducted in the villages of the foci and the surrounding villages. In the second layer, chemoprophylaxis and ACD were conducted mainly in the townships of the foci and the surrounding townships. In the third layer, preventive medication and health education were conducted in Sanya and the associated counties.

\section{Data collection and statistical analysis}

Basic and detailed malaria case information were gathered from the Infectious Diseases Information Reporting Management System (IDIRMS) and the information of 1-3-7 were gathered from Parasitic Diseases Information Reporting Management System (PDIRMS )[15]. The historical malaria information data before 2004 were gathered from reports or archives. Detailed TLS information on the implementation for blocking $P$. malariae transmission from 2015 to 2018 was obtained from the annual reporting reports or archives of the local CDC. The population data and other relevant information on the villages were obtained from government annual reports or archives. The data were entered by Microsoft Office Excel 2007 for collection and analysis by SPSS.

\section{Results}

\section{History of malaria surveillance in Sanya and in Hainan}

Hainan had malaria endemic areas of $P$. falciparum and $P$. vivax. Since 1954, a parasite survey of local residents (PSLR), which acted as a type of surveillance for ACD, was conducted in Hainan Province every year. From PSLR recordings, 10 cases (Cl: 2-42) of $P$. malariae were found every year in Hainan Province before 1964. However, Sanya also conducted PSLR but found only $P$. falciparum malaria and $P$. vivax since 1959[16]. From IDIRMS, 27 malaria cases were reported in Sanya from 2009 to 2018, including 6 cases of $P$. falciparum malaria, 10 cases of $P$. vivax malaria, 6 cases of $P$. malariae malaria, 1 case of $P$. ovale malaria, and 4 cases of unclassified malaria. During the malaria elimination phase from 2011 to 2018 , a total of 14 cases of malaria were reported in Sanya city, including 8 indigenous cases and 6 imported cases. The indigenous cases reported in 2011 were $P$. vivax. No cases were reported from 2012 to 2014 and 2017[17]. In 2015, 6 indigenous cases of $P$. malariae were found (Fig. 1).

\section{Established epidemic response for forest-goers in TLS in 2015}


Three villages in Gaofeng town of Sanya city were selected as the first layer, including Baolong, Lixin and Zhanan. Seven villages in Xinzheng Town, Baoting County, were also selected as the first layer, including shirang, maowen, xinzheng, maopeng, baodao, shenna and nangai; All of the residents were targeted except for migrants and were not suitable for users, with active case detection (ACD). 1,823 blood slides and PCR screening from villages in Baoting and Sanya were conducted on all residents. Three positive samples were found from blood samples, and thirteen positive samples were found from PCR. After cross-checking, four cases were identified and other false positives were ruled out, including Case 2, 3, 4 and Case 6 . Case 5 was found when he went to the hospital voluntarily. they were confirmed to be infected with $P$. malariae. In the second layers, ten townships were chosen, and passive case detection (PCD) and active case detection (ACD) for forest-goers were conducted mainly in Gaofeng townships of the foci and surrounding townships, including Gaofeng, Yucai in Sanya city, Daan, Zhizhong in Ledong County, Xiangshui, Maogan, Nanlin, Sandao, Xinzhen in Baoting County and Changhao in Wuzhishan City. 7,831 blood slides were screened from target townships and tests conducted on all forest-goers. In the third layer, 12 counties or cities were selected for health education, and passive case detection was conducted mainly around Sanya and the associated counties for forest-goers, including Ledong, Baoting, Wuzhishan, Dongfang, Lingshui, Qiongzhong, Baisha, Changjiang, Wanning, Qionghai, Tunchang and Danzhou. 95,350 blood slides were screened from target townships, and tests were conducted on all forest-goers (Table 1). Meanwhile, vector surveillance and control were also conducted to support foci disposals and to minimize the possibility of transmission (Table 2). 
Table 1

Three-layer strategy conducted in epidemic response in 2015 and intervention from 2016 to 2018

\begin{tabular}{|c|c|c|c|c|c|c|c|c|c|c|c|c|c|}
\hline \multirow[t]{4}{*}{ Layers } & \multirow{4}{*}{$\begin{array}{l}\text { Names of } \\
\text { counties or } \\
\text { cities }\end{array}$} & \multirow{4}{*}{$\begin{array}{l}\text { Names of } \\
\text { township }\end{array}$} & \multirow{4}{*}{$\begin{array}{l}\text { Names of } \\
\text { village }\end{array}$} & \multicolumn{10}{|c|}{ Three-layer strategy (TLS) } \\
\hline & & & & \multicolumn{4}{|c|}{ Epidemic response in 2015} & \multicolumn{6}{|c|}{ Strengthened intervention from 2016 to 2018} \\
\hline & & & & \multicolumn{2}{|l|}{ PCD } & \multicolumn{2}{|l|}{ ACD } & \multirow{2}{*}{$\begin{array}{l}2016 \\
\text { MPD }\end{array}$} & \multirow{2}{*}{$\begin{array}{l}2017 \\
\text { MPD }\end{array}$} & \multirow{2}{*}{$\begin{array}{l}2018 \\
\text { MPD }\end{array}$} & \multirow{2}{*}{$\begin{array}{l}\begin{array}{l}\text { ACD } \\
\text { in }\end{array} \\
\text { FG }\end{array}$} & \multirow[t]{2}{*}{$\mathrm{CP}$} & \multirow[t]{2}{*}{$\%$} \\
\hline & & & & Ne. & Pos. & Ne. & Pos. & & & & & & \\
\hline \multirow{10}{*}{$\begin{array}{l}1^{\text {st }} \\
\text { Layer }\end{array}$} & \multirow[t]{3}{*}{ Sanya } & \multirow[t]{3}{*}{ Gaofeng } & Baolongt & 67 & 0 & 400 & 0 & 50 & 51 & 29 & 256 & 216 & $84.4 \%$ \\
\hline & & & Lixint & 74 & 0 & 558 & 4 & 55 & 46 & 34 & 827 & 663 & $80.2 \%$ \\
\hline & & & Zhanant & 35 & 1 & 684 & 0 & 29 & 46 & 58 & 1030 & 946 & $91.8 \%$ \\
\hline & \multirow[t]{7}{*}{ Baoting } & \multirow[t]{7}{*}{ xinzheng } & shirang & 58 & 0 & 17 & 0 & 107 & 150 & 3 & 116 & 116 & $100.0 \%$ \\
\hline & & & maowen & 45 & 0 & 5 & 0 & 37 & 17 & 0 & 173 & 171 & $98.8 \%$ \\
\hline & & & xinzheng & 39 & 0 & 8 & 0 & 288 & 14 & 0 & 275 & 250 & $90.9 \%$ \\
\hline & & & maopeng & 53 & 0 & 7 & 0 & 50 & 29 & 1 & 103 & 96 & $93.2 \%$ \\
\hline & & & baodao & 43 & 0 & 11 & 0 & 39 & 16 & 0 & 57 & 54 & $94.7 \%$ \\
\hline & & & shenna & 41 & 0 & 27 & 0 & 35 & 11 & 0 & 116 & 116 & $100.0 \%$ \\
\hline & & & nangai & 41 & 0 & 106 & 0 & 137 & 184 & 0 & 173 & 171 & $98.8 \%$ \\
\hline \multirow{11}{*}{$\begin{array}{l}2^{\text {nd }} \\
\text { Layer }\end{array}$} & \multirow[t]{2}{*}{ Sanya } & Gaofeng* & & 1216 & 0 & 0 & 0 & 1071 & 178 & 165 & 0 & 0 & 0 \\
\hline & & Yucai & & 1109 & 0 & 553 & 0 & 602 & 1109 & 1219 & 1297 & 1265 & $97.5 \%$ \\
\hline & \multirow[t]{3}{*}{ ledong } & Daan & & 83 & 0 & 317 & 0 & 550 & 550 & 548 & 5 & 5 & $100.0 \%$ \\
\hline & & Zhizhong & & 548 & 0 & 32 & 0 & 702 & 714 & 664 & 378 & 375 & $99.2 \%$ \\
\hline & & Baoguo & & 82 & 0 & 447 & 0 & 513 & 552 & 520 & 30 & 24 & $80.0 \%$ \\
\hline & Baoting & Xiangshui & & 331 & 0 & 103 & 0 & 475 & 474 & 471 & 415 & 375 & $90.4 \%$ \\
\hline & & Maogan & & 350 & 0 & 0 & 0 & 549 & 414 & 588 & 1381 & 341 & $24.7 \%$ \\
\hline & & Nanlin & & 600 & 0 & 258 & 0 & 747 & 686 & 610 & 478 & 478 & $100.0 \%$ \\
\hline & & Xinzheng & & 742 & 0 & 0 & 0 & 797 & 557 & 425 & 707 & 707 & $100.0 \%$ \\
\hline & & Sandao & & 740 & 0 & 0 & 0 & 708 & 554 & 481 & 427 & 414 & $97.0 \%$ \\
\hline & Wuzhishan & changhao & & 320 & 0 & 0 & 0 & 317 & 444 & 302 & 371 & 360 & $97.0 \%$ \\
\hline $3^{\text {rd }}$ & Sanya* & & & 11039 & 0 & 15 & 0 & 10815 & 8183 & 9186 & 0 & 0 & 0 \\
\hline & Ledong & & & 4261 & 0 & 4332 & 0 & 8372 & 8533 & 8662 & 191 & 178 & $93.2 \%$ \\
\hline & Baoting* & & & 5529 & 0 & 142 & 0 & 7371 & 4878 & 5330 & 0 & 0 & 0 \\
\hline & Wuzhishan & & & 2120 & 0 & 885 & 0 & 2972 & 3009 & 2472 & 726 & 712 & $98.1 \%$ \\
\hline & Dongfang & & & 9496 & 0 & 0 & 0 & 9308 & 9755 & 9462 & 489 & 473 & $96.7 \%$ \\
\hline & Lingshui & & & 5359 & 0 & 1747 & 0 & 7421 & 7171 & 7477 & 833 & 808 & $97.0 \%$ \\
\hline & Qiongzhong & & & 3427 & 0 & 2640 & 0 & 5965 & 6724 & 5975 & 427 & 425 & $99.5 \%$ \\
\hline & Baisha & & & 6828 & 0 & 588 & 0 & 7365 & 7342 & 6354 & 207 & 207 & $100.0 \%$ \\
\hline & Changjiang & & & 2294 & 0 & 2923 & 0 & 5226 & 5172 & 5334 & 1418 & 1417 & $99.9 \%$ \\
\hline & Waning & & & 9354 & 0 & 1115 & 0 & 13616 & 11287 & 18427 & 3732 & 3366 & $90.2 \%$ \\
\hline & Qionghai & & & 5582 & 0 & 1624 & 0 & 8030 & 7604 & 6742 & 2016 & 1952 & $96.8 \%$ \\
\hline & Tunchang & & & 1969 & 0 & 1626 & 0 & 3523 & 3598 & 3098 & 0 & 0 & 0 \\
\hline & Danzhou & & & 10297 & 0 & 158 & 0 & 9602 & 93687 & 9111 & 325 & 319 & $98.2 \%$ \\
\hline
\end{tabular}

†Baolong village consists 4 units, Hongqi, Zhatao, Zhaban, Xianjin; Zhanan village consists of 8 units, Nanjin, Hongxing, Jiayue, xincun, Baotu, Ganyou, Lingqu; Lixin village consists of 4 units, Zhayun, Zhaka, Zhaye, Zhachu, Zhaye ,xincun;

* The data has been shown in the previous layer.

MPD: malaria parasite detection;FG:Forest goer;CP: chemoprevention;ACD: active case detection; PCD:passive case detection 


\begin{tabular}{|c|c|c|c|c|c|c|c|c|c|c|c|c|c|}
\hline \multirow[t]{4}{*}{ Layers } & \multirow{4}{*}{$\begin{array}{l}\text { Names of } \\
\text { counties or } \\
\text { cities }\end{array}$} & \multirow{4}{*}{$\begin{array}{l}\text { Names of } \\
\text { township }\end{array}$} & \multirow{4}{*}{$\begin{array}{l}\text { Names of } \\
\text { village }\end{array}$} & \multicolumn{10}{|c|}{ Three-layer strategy (TLS) } \\
\hline & & & & \multicolumn{4}{|c|}{ Epidemic response in 2015} & \multicolumn{6}{|c|}{ Strengthened intervention from 2016 to 2018} \\
\hline & & & & \multicolumn{2}{|l|}{ PCD } & \multicolumn{2}{|l|}{ ACD } & \multirow{2}{*}{$\begin{array}{l}2016 \\
\text { MPD }\end{array}$} & \multirow{2}{*}{$\begin{array}{l}2017 \\
\text { MPD }\end{array}$} & \multirow{2}{*}{$\begin{array}{l}2018 \\
\text { MPD }\end{array}$} & \multirow{2}{*}{$\begin{array}{l}\text { ACD } \\
\text { in } \\
\text { FG }\end{array}$} & \multirow[t]{2}{*}{$\mathrm{CP}$} & \multirow[t]{2}{*}{$\%$} \\
\hline & & & & Ne. & Pos. & Ne. & Pos. & & & & & & \\
\hline Total & & & & 84172 & 1 & 21328 & 4 & 107044 & 183739 & 103748 & 18979 & 17000 & $89.6 \%$ \\
\hline \multicolumn{14}{|c|}{$\begin{array}{l}\text { †Baolong village consists } 4 \text { units, Hongqi, Zhatao, Zhaban, Xianjin; Zhanan village consists of } 8 \text { units, Nanjin, Hongxing, Jiayue, xincun, Baotu, Ganyou, } \\
\text { Lingqu; Lixin village consists of } 4 \text { units, Zhayun, Zhaka, Zhaye, Zhachu, Zhaye ,xincun; }\end{array}$} \\
\hline \multicolumn{14}{|c|}{ * The data has been shown in the previous layer. } \\
\hline \multicolumn{14}{|c|}{ MPD: malaria parasite detection;FG:Forest goer;CP: chemoprevention;ACD: active case detection; PCD:passive case detection } \\
\hline
\end{tabular}

Table 2

Vector Surveillance and control from 2015 to 2018

\begin{tabular}{|c|c|c|c|c|c|c|c|c|c|c|c|c|c|c|c|}
\hline \multirow{3}{*}{$\begin{array}{l}\text { Names of } \\
\text { counties or } \\
\text { cities }\end{array}$} & \multicolumn{15}{|c|}{ Vector Surveillance } \\
\hline & \multicolumn{4}{|l|}{2015} & \multicolumn{4}{|l|}{2016} & \multicolumn{4}{|l|}{2017} & \multicolumn{3}{|l|}{2018} \\
\hline & $\begin{array}{l}\text { An. } \\
\text { minus }\end{array}$ & $\begin{array}{l}\text { An. } \\
\text { dirus }\end{array}$ & $\begin{array}{l}\text { An. } \\
\text { sinensis }\end{array}$ & others & $\begin{array}{l}\text { An. } \\
\text { minus }\end{array}$ & $\begin{array}{l}\text { An. } \\
\text { dirus }\end{array}$ & $\begin{array}{l}\text { An. } \\
\text { sinensis }\end{array}$ & others & $\begin{array}{l}\text { An. } \\
\text { minus }\end{array}$ & $\begin{array}{l}\text { An. } \\
\text { dirus }\end{array}$ & $\begin{array}{l}\text { An. } \\
\text { sinensis }\end{array}$ & others & $\begin{array}{l}\text { An. } \\
\text { minus }\end{array}$ & $\begin{array}{l}\text { An. } \\
\text { dirus }\end{array}$ & $\begin{array}{l}\text { An. } \\
\text { sine }\end{array}$ \\
\hline Sanya & 1 & 0 & 154 & 2337 & 1 & 2 & 368 & 50 & 0 & 0 & 548 & 140 & 0 & 0 & 187 \\
\hline Ledong & 0 & 0 & 164 & 283 & 0 & 0 & 182 & 143 & 0 & 0 & 105 & 60 & 0 & 0 & 83 \\
\hline Baoting & 0 & 0 & 0 & 15 & 0 & 0 & 319 & 204 & 0 & 0 & 131 & 352 & 0 & 0 & 9 \\
\hline Wuzhishan & 2 & 18 & 74 & 29 & 2 & 19 & 49 & 5 & 0 & 34 & 10 & 2 & 2 & 21 & 21 \\
\hline Dongfang & 0 & 0 & 92 & 296 & 0 & 0 & 11 & 85 & 0 & 0 & 181 & 423 & 0 & 0 & 207 \\
\hline Lingshui & 0 & 0 & 95 & 273 & 0 & 0 & 241 & 802 & 0 & 0 & 236 & 675 & 0 & 0 & 88 \\
\hline Qiongzhong & 0 & 0 & 357 & 226 & 0 & 0 & 119 & 17 & 0 & 0 & 61 & 16 & 0 & 0 & 36 \\
\hline Baisha & 3 & 19 & 910 & 1126 & 9 & 0 & 242 & 409 & 0 & 0 & 418 & 126 & 1 & 0 & 224 \\
\hline Changjiang & 100 & 0 & 327 & 1137 & 0 & 40 & 116 & 268 & 1 & 3 & 93 & 212 & 1 & 0 & 9 \\
\hline Wanning & 0 & 0 & 97 & 214 & 0 & 0 & 116 & 164 & 0 & 0 & 94 & 154 & 0 & 0 & 65 \\
\hline Qionghai & 0 & 0 & 0 & 0 & 1 & 0 & 663 & 185 & 1 & 0 & 107 & 312 & 23 & 0 & 53 \\
\hline Tunchang & 17 & 0 & 1249 & 2927 & 9 & 0 & 1081 & 3647 & 19 & 0 & 2253 & 3110 & 0 & 0 & 470 \\
\hline Danzhou & 154 & 0 & 73 & 11 & 37 & 0 & 124 & 154 & 1 & 0 & 108 & 10 & 0 & 0 & 120 \\
\hline Total & 277 & 37 & 3592 & 8874 & 59 & 61 & 3631 & 6133 & 22 & 37 & 4345 & 5592 & 27 & 21 & $157 \varepsilon$ \\
\hline
\end{tabular}

*The data of IRS didn't showed and IRS implemented only in Sanya for disposal six focis.

\section{Strengthened intervention on forest-goers in TLS from 2016 to 2018}

Target villages between 2016 and 2018 were included in the epidemic response strategy for forest-goers in 2015, but residents adopted the number of residents at that time. Piperaquine phosphate was selected as a chemoprophylactic drug. In March from 2016 to 2018 , residents in the targeted villages were given a total dose of $1200 \mathrm{mg}$ orally before bedtime for adults within 3 days, from 2016 to 2018. Starting in April from 2016 to 2018 , forest-goers were administered $600 \mathrm{mg}$ orally for adults before bedtime within a day. Doses for children decreased by weight or age. Villagers, including forest-goers, were required to sign informed consent before administration. At the same time, active case detection (ACD) should be conducted while seeking forest-goers. If fever is found in forest-goers, examination for malaria must be performed, including microscopy.(Fig. 2)

In the first layer, chemoprophylaxis and ACD were conducted on residents in the villages of the foci and the surrounding villages, and $89.5 \%$ residents accepted chemoprophylaxis. In the second layer, chemoprophylaxis and ACD while seeking forest-goers were conducted mainly in the townships of the foci and the surrounding townships. Of the 5,489 people who were forest-goers, $79.1 \%$ received chemoprophylaxis while seeking forest-goers. In the third layer, preventive medication and health education were conducted in Sanya and the associated counties for forest-goers. Of the 10,364 people who belonged to forest-goers, $89.6 \%$ received chemoprophylaxis while seeking forest-goers. Meanwhile, the training of clinicians, public health and laboratory personnel was strengthened to improve the capacity to respond to the epidemic from 2015 to 2018 (Table 3). 
Table 3

Training in 13 counties or cities for response and intervention from 2015 to 2018

\begin{tabular}{|c|c|c|c|c|c|c|c|c|c|c|c|c|}
\hline \multirow[t]{2}{*}{ Names of counties or cities } & \multicolumn{4}{|c|}{ Clinician } & \multicolumn{4}{|c|}{ Public Health Doctor } & \multicolumn{4}{|c|}{ Laboratory Technicians } \\
\hline & 2015 & 2016 & 2017 & 2018 & 2015 & 2016 & 2017 & 2018 & 2015 & 2016 & 2017 & 2018 \\
\hline Sanya & 23 & 18 & 20 & 24 & 36 & 24 & 20 & 22 & 29 & 18 & 26 & 19 \\
\hline Ledong & 25 & 26 & 46 & 0 & 51 & 68 & 34 & 23 & 25 & 26 & 46 & 23 \\
\hline Baoting & 28 & 23 & 24 & 17 & 83 & 43 & 72 & 20 & 16 & 17 & 19 & 15 \\
\hline Wuzhishan & 9 & 11 & 10 & 0 & 10 & 10 & 10 & 23 & 10 & 10 & 6 & 0 \\
\hline Dongfang & 24 & 21 & 22 & 27 & 42 & 42 & 22 & 27 & 23 & 21 & 23 & 22 \\
\hline Lingshui & 21 & 46 & 56 & 22 & 45 & 66 & 56 & 56 & 42 & 25 & 22 & 22 \\
\hline Qiongzhong & 105 & 0 & 25 & 24 & 0 & 0 & 0 & 0 & 2 & 0 & 27 & 4 \\
\hline Baisha & 26 & 85 & 24 & 0 & 42 & 54 & 0 & 0 & 31 & 38 & 26 & 0 \\
\hline Changjiang & 10 & 16 & 27 & 0 & 21 & 21 & 30 & 33 & 14 & 25 & 30 & 25 \\
\hline Waning & 15 & 100 & 24 & 23 & 335 & 190 & 24 & 24 & 0 & 32 & 22 & 20 \\
\hline Qionghai & 10 & 0 & 73 & 27 & 70 & 60 & 51 & 21 & 34 & 0 & 20 & 17 \\
\hline Tunchang & 25 & 11 & 19 & 44 & 21 & 20 & 15 & 45 & 18 & 14 & 22 & 38 \\
\hline Danzhou & 28 & 18 & 17 & 0 & 106 & 34 & 17 & 23 & 47 & 32 & 17 & 0 \\
\hline Total & 349 & 375 & 387 & 208 & 862 & 632 & 351 & 317 & 291 & 258 & 306 & 205 \\
\hline
\end{tabular}

\section{Case reports and epidemiological characteristics of this outbreak under TLS guideline}

A total of 6 P. malariae cases occurred in Sanya city in 2015 under TLS guideline. All cases except for the index case (case 1) were reported within 1 day through IDIRMS. From PDIRMS, None of the 6 patients had a history of travelling abroad, previous malaria or blood transfusion. Six cases were regarded as indigenous cases of $P$. malariae. All of the reported cases were confirmed by microscopy and sequencing. These cases were reported between September and November: 3 cases in September, 1 case in October and 1 case in November. The age group (19-40 years of age) belonged to the younger-middle age group. They were all males and farmers in occupational categories. The total indigenous cases were reported at the Gaofeng township level ( $\mathrm{N}=6,100 \%)$ or in 3 villages. Two cases(Case 1 and 5) were found by passive case detection (PCD), but 4 cases were found by active case detection (ACD) in their villages (Table 4). We also collected information about the outpatient visiting behaviors and found that $100 \%$ of patients visited local village clinics after experiencing fever symptoms. However, only $16 \%$ (case 1 ) of patients were diagnosed with malaria in county-level hospitals, although $33 \%$ (cases 1 and 5 ) continued to higherlevel hospitals, including township-level or/and county-level hospitals. 
Table 4

Demographic epidemic characteristics of cases

\begin{tabular}{|c|c|c|}
\hline & No. Cases $(\mathrm{N}=6)$ & $(\%)$ \\
\hline \multicolumn{3}{|l|}{ Ages } \\
\hline$<20$ & 1 & $16.7 \%$ \\
\hline $20-30$ & 2 & $33.3 \%$ \\
\hline $30-40$ & 3 & $50 \%$ \\
\hline \multicolumn{3}{|l|}{ Gender } \\
\hline male & 6 & $100 \%$ \\
\hline \multicolumn{3}{|l|}{ Address } \\
\hline Gaofeng towns & 6 & $100 \%$ \\
\hline \multicolumn{3}{|l|}{ Village } \\
\hline Baolong & 1 & $16.7 \%$ \\
\hline Lixin & 4 & $66.7 \%$ \\
\hline Zhanan & 1 & $16.7 \%$ \\
\hline Number of forest goer & 5 & $83.3 \%$ \\
\hline \multicolumn{3}{|l|}{ Source of cases } \\
\hline PCD & 2 & $33.3 \%$ \\
\hline ACD & 4 & $66.7 \%$ \\
\hline \multicolumn{3}{|l|}{ 1-3-7 implement } \\
\hline Report within $24 \mathrm{~h}$ & 5 & $83.3 \%$ \\
\hline Confirmation and epidemical investigation within 3 days & 6 & $100 \%$ \\
\hline Foci classification and response within 7 days & 6 & $100 \%$ \\
\hline
\end{tabular}

From the results of the epidemic survey, 5 patients had a history of night sleeping in mountains before the onset of fever and were classified as forest-goers. However, one case (case 6) had no above mentioned history before the onset of fever. A total of 5 cases (Case1, Case2, Case3, Case4, Case5) had a history of collecting bodhi fruit (Daemonoropus margaritae Becc varpalawanica) or honey in the mountains in almost all months of the year. Case 1, who coworked with cases 2, 3 and 4, climbed mountains and slept overnight to collect bodhi fruit, and case 5, who coworked with the other 2 persons, did so also. However, Case 6 stayed in villages and had no history of staying in mountains overnight. From Case 1 to Case 5 , infection with $P$. malariae was associated with the behaviour of overnights in the mountains. Moreover, infection also occurred in the village from case 6 (Fig. 3).

\section{Infection source}

A total of 6 cases were found in this epidemic, among which 5 cases belonged to forest-goers, who had a history of overnight sleeping in the mountains, and 1 case did not belong to forest-goers and had no history of overnight sleeping in mountains. At present, there are three possible sources of infection in mosquito-borne malaria cases: 1) transmission caused by a long incubation period. 2) transmission caused by imported sources of infection and subsequent reintroduction. 3) transmission from an animal host in forests. Based on the epidemic characteristics of these cases, especially age characteristics, the second is more likely, the third is possible, and the first is almost impossible.

\section{Discussion}

Malaria infection in forest-goers has also been an important and difficult point with regard to malaria elimination in Hainan. Forest-goers were the main group of malaria infections in Hainan, mostly due to the abundant forest products combined with human behaviour. Abundant forest products, such as wood, honey and wild animals, have attracted attention from local residents and mobile populations [18]. The behaviour of these people, especially going overnight without nets, leads to malaria infection. In malaria control stage, an investigation in Nanqiao, Wanning City showed that overnight behaviour in mountains, the low usage rate of mosquito nets and the lack of malaria prevention knowledge were the main factors that affected the epidemics and the control of malaria [19, 20]. In the stage of elimination of malaria, this outbreak was a typical case of malaria transmission caused by forest-goers, and five of them had a history of staying overnight in mountains, although they were infected with P. malariae, which was first discovered in Sanya, Hainan.

Based on this outbreak and other outbreaks in other parts of China, outbreaks still occur during the malaria elimination phase[21, 22], although its much less than during the control stage. Innovative TLSs were invented and first applied in this outbreak, and Case 6 was importantly detected as an asymptomatic carrier. If only the 1-3-7 approach had been implemented, Cases 2-4 associated with Case 1 and two persons associated with Case 5 would be tested. Comparing the results of the 1-3-7 approach to the results of this study, TLS improved the case detection ability and detected potential sources of infection, 
such as asymptomatic carriers and cases who did not seek medical treatment. TLS may also be suitable for outbreaks in the screening of unexplained indigenous cases or introduced cases in countries and regions that are undergoing malaria elimination and for current malaria outbreak areas.

From the results of the disposal outbreak in this study using TLS, the spread scope of the epidemic was further estimated, and four cases were distributed in different villages, but they belonged to the same township as GaoFeng township. No malaria cases have been found outside Sanya city. This information may be further applied in the management of high-risk groups and different intervention measures adopted in different layers, which are similar to the current strategies against COVID-19 in China[23].

This outbreak was caused by P. malariae and was highly likely an imported-introduced case. Sanya is a tourist city with a large number of migrant people, including people from abroad, and An. minimus, which is the main effective vector of malaria in Hainan, also exists in Sanya. According to epidemiological investigation data and the characteristics of $P$. malariae transmission, the present study concluded that there was a high possibility of human-to-mosquito-tohuman transmission in forest-goers. From the current release of $P$. malariae cases, primarily from Guangdong and Shanghai [24, 25], these cases indicate that $P$. malariae has a long incubation period and may cause a life-long infection, with or without recurrent fever. However, these cases were in older age groups rather than young-to-middle age groups, which is the largest difference from this outbreak. The human-monkey mode of transmission is impossible because only 5 cases were found at this time. There have been no subsequent cases in the mountains, although forest-goers have existed since 2015 . However, we cannot exclude macaque infections with $P$. malariae. All of the malaria parasites in this outbreak were identified as $P$. malariae, not $P$. simium, which led to zoonosis in forest-goers in Brazil[26]. Cities or counties in central Hainan province, where An. dirus and An. minimus exist, are actively developing tourism resources for economic development [26]. It is more challenging to prevent the retransmission of imported malaria, and forest-goers remain a high-risk group in preventing the retransmission of malaria.

\section{Conclusions}

TLS is suitable for outbreak by indigenous case of unknown causes or for sporadic cases with defects in epidemic information, while 1-3-7 is suitable for sporadic cases which have clearly epidemic clues, such as imported cases and coworker with imported cases. Moreover, TLS could be one of the major achievements after 1-3-7 approach, targeting outbreak in elimination malaria. The innovative TLS is effective in forest-goers in Hainan in this study and can be applied to southeast Asia, where An. dirus and An. minimus are transmitted, to address malaria elimination and control.

\section{Abbreviations}

CDC: Center for Diseases Control and Prevention;

IRS: Indoor residual spraying;

ITN: Insecticide-treated bed nets;

MDA: Mass drug administration;

TLS: Three-Layer Strategy;

LLINs: long lasting insecticide nets.

FG: forest goer;

NMEP: National Malaria Elimination Programme;

JPCS: Joint prevention and control strategies;

ACD: active case detection;

PCD: passive case detection;

IDIRMS: Infectious Diseases Information Reporting Management System;

PDIRMS: Parasitic Diseases Information Reporting Management System;

PSLR: parasite survey of local residents;

MPD: malaria parasite detection;

\section{Declarations}

\section{Ethical Approval and Consent to participate}

This study is a retrospective study based on the treatment of malaria outbreak in Sanya in 2015. In this study, Blood slides or blood spot samples in filter papar collected from local residents and forest goers were only used for medical malaria detection. In order to prevent the transmission of malaria, informed consent was subsequently signed for those taking piperaquine, informing them of the medication procedure, adverse reactions and the purpose of the medication. The above actions were approved by Hainan Provincial Health and family Planning Commission (ID:201536,201603,201714,201807).

Page $10 / 14$ 


\section{Consent for publication}

This paper is published with the authority from the Hainan Provincial Center for Disease Control and Prevention.

\section{Availability of data and materials}

All the relevant datasets supporting the conclusions of this article are included within the article.

\section{Competing interests}

The authors declare no competing interests.

\section{Funding}

This work was supported by Bill \& Melinda Gates Foundation: No. INV-018913 and Hainan Provincial Basic and Applied Basic Research Program (Natural Science Foundation) for High-level Talents in 2019(2019RC394)

\section{Authors' contributions}

YCL conceived the paper, analysed results and wrote the first version of the manuscript. WZ, FM, XMH, RQC, YJH, WZH, HZX, RSY, SLH, JZ, XDW, GYW, YL, HSL, ZCZ, JW, GSW supported data collection. GZW, BY, YC supported interpretation of results and revision of the manuscript. All authors read and approved the final manuscript.

\section{Acknowledgements}

We acknowledge the contributions of all the officials, professionals and people involved in controlling this outbreak. Moreover, Xiaonong Zhou and Jianhai Yin gave guide to this manuscript.

\section{References}

1. Wei S, Lin S, Yang C. The 50-Year History of Health Service Development in Hainan Province from 1950 to 2000. Haikou: Nanfang Press, 2007

2. Lin M, Chen S, Deng J, Si Y, Zhong H, Huang M, Chen X, Cao R. Investigation on malaria infection among residents of FOREST GOERS accommodation in high malaria area of Hainan Province. Hainan Med J 1993; 4: 8-9

3. Jin Y, Wang S, Meng F, Zhuo K, Huade, Lin C, Fu H, Huang Y. Investigation on malaria among residents and overnight people in Wuzhishan city. Chin J Trop Med 2003; 6: 738-739

4. He CH, Hu XM, Wang GZ, Zhao W, Sun DW, Li YC, Chen CX, Du JW, Wang SQ. Eliminating Plasmodium falciparum in Hainan, China: a study on the use of behavioural change communication intervention to promote malaria prevention in mountain worker populations. Malar J 2014; 13 : 273

5. Lan CX, Cai XZ, Zeng LH, Lin SG, Lan XH, Cai ZF, Liang ZT, Lin MH, Huang MS, Chen X. Study on prevention and control of malaria infection among mountain population in Nanqiao area of Hainan Province. Hainan Med J 1998; 1: 1

6. Lin C, Lin S, Fu Z, Ji W, Li H. Investigation and analysis of malaria control among FOREST GOERS population in malaria area of Ledong County. Chin J Trop Med 2005; 2: 257-335

7. Lin M, Weng S. Progress on malaria control in 60 years in Nanqiao Pilot project and progress in the implementation of global fund projects against malaria. Chin J Trop Med 2013; 132: 245-248

8. Fan N, Zeng W. Malaria elimination action plan launched in Hainan. Hainan Daily, 2010-03-20B06.

9. Lin C, Chen Z, Wang S, Luo P, Wu D, Zheng A, Wei J. Analysis and management of a rare malaria case of $P$. malariae in sanya, Hainan Province. Chin J Trop Med 2016; 165: 481-484

10. Zhou SS, Zhang SS, Zhang L, Rietveld AE, Ramsay AR, Zachariah R, Bissell K, Van Den Bergh R, Xia ZG, Zhou XN, Cibulskis RE. China's 1-3-7 surveillance and response strategy for malaria elimination: is case reporting, investigation and foci response happening according to plan? Infect Dis Poverty $2015 ; 4$ : 55

11. Ma K. China has been Certified by WHO to Eliminate Malaria, and Hainan is One of the Four Provinces to be Certified On-Site. Hainan Daily 2021; July 27: B06

12. Wang SQ. The seventy years of malaria from hyperendemicity to elimination in Hainan. Chin J Trop Med 2019; 198: 707-718

13. Wang GZ, Wang SQ, Hu M, Zhu QX, Zeng W, Cai HL, Li YC, Meng F. A retrospective analysis on the effectiveness of twenty years' malaria joint control in Hanan province. Chin J Trop Med 2013; 1311: 1339-1342

14. Li YC, Wang GZ, Sun DW, Meng F, Lin SG, Hu XM, Wang SQ. A case of Plasmodium ovale wallikeri infection in a Chinese worker returning from West Africa. Korean J Parasitol 2013; 5: 557-562

15. Dai S, Zhu M, Wu H, Zhang Y, Wang Z, Zhang C, Ma X, Jiang L. From malaria elimination to post-elimination: a 10-year surveillance data study in Shanghai. Malar J 2021; 201: 199

16. Institute of Parasitic Diseases of Hainan Administrative Region. Malaria Control Research Data in Hainan Island 1950-1983, 1985, P2-120

17. Xie Y, Ma C, Zeng W, Wang G, Meng F, Li Y, Huang Y, Xing C, Wang J. Hainan province in 2011-2016 epidemiological characteristics of imported malaria. China Trop Med 2018; 193: 252-255 
18. Wu K, Chen W, Tang L, Deng D, Lin M, Cai X, Pan Y, Gu Z, Yan W, Huang M, Zhu W, Sheng H, Chen X. Study on the characteristics of lodging behavior and its relationship with malaria infection among $\mathrm{Li}$ and Miao ethnic groups in high malaria mountainous area of Hainan Province. Chin $\mathrm{J}$ Parasitol Parasitosis 1995; 4: 17-21

19. Wu K, Tang L, Chen W, Liu D, Lin M, Gu Z, Lan C, He Y, Wang Z, Chen G, Cai X, Deng D. Epidemiological characteristics of malaria in mountainous areas of Hainan. Chin J Parasit Dis 1998; 4: 3-7

20. Chen W, Shi P, Wu K, Deng D, Tang L, Cai X, Gu Z, Si Y, Lin M, Yan W. Impact of socio-economic factors on malaria prevalence in Hainan province by unconditional logistic multiple regression analysis. Chin J Parasit Dis 1995; 3: 161-164

21. Sun YW, YU DM, Chen J, Li X, Wang B, Wang ZJ, Mao LL, Yao WQ. Analysis of two cases of Plasmodium vivax malaria in dandong city, liaoning province. Chin J public health 2017; 332:314-316

22. Feng XY, Shi WQ, Li JL, Chen JS, Li ZX, Xia ZG. Investigation of malaria vectors in Longhui county, Hunan province. Chin J pathog biol 2020;1503:317321.

23. Lei ZL. What is the difference between containment area, control area or prevention area. 2021. https://xw.qq.com/cmsid/20211023A04QQX00?f=newdc. Accessed 16 Sep 2021.

24. Pan B, Ruan CW, Pei FQ, Lin GQ, Cen YZ, Zhang ZX. Diagnosis and treatment analysis of a case of malariae malaria with long latent period. South China J Prev Med 2016; 421: 64-67

25. Cao S, Wang L, Zhu M, Cai L. Traceability investigation of a P. malariae case in Shanghai. Chin J Parasitol Parasit Dis 2016; 342: 150-153

26. Brasil P, Zalis MG, de Pina-Costa A, Siqueira AM, Júnior CB, Silva S, Areas ALL, Pelajo-Machado M, De Alvarenga DAM, Da Silva Santelli ACF. Outbreak of human malaria caused by Plasmodium simium in the Atlantic forest in Rio de Janeiro: a molecular epidemiological investigation. Lancet Glob Health 2017; 510: e1038-e1046

27. Shi X. Measurement and evaluation of tourism ecological coupling degree under the construction of Hainan free trade port. China Bus Theory $2021 ; 20$ : $40-42$

\section{Figures}
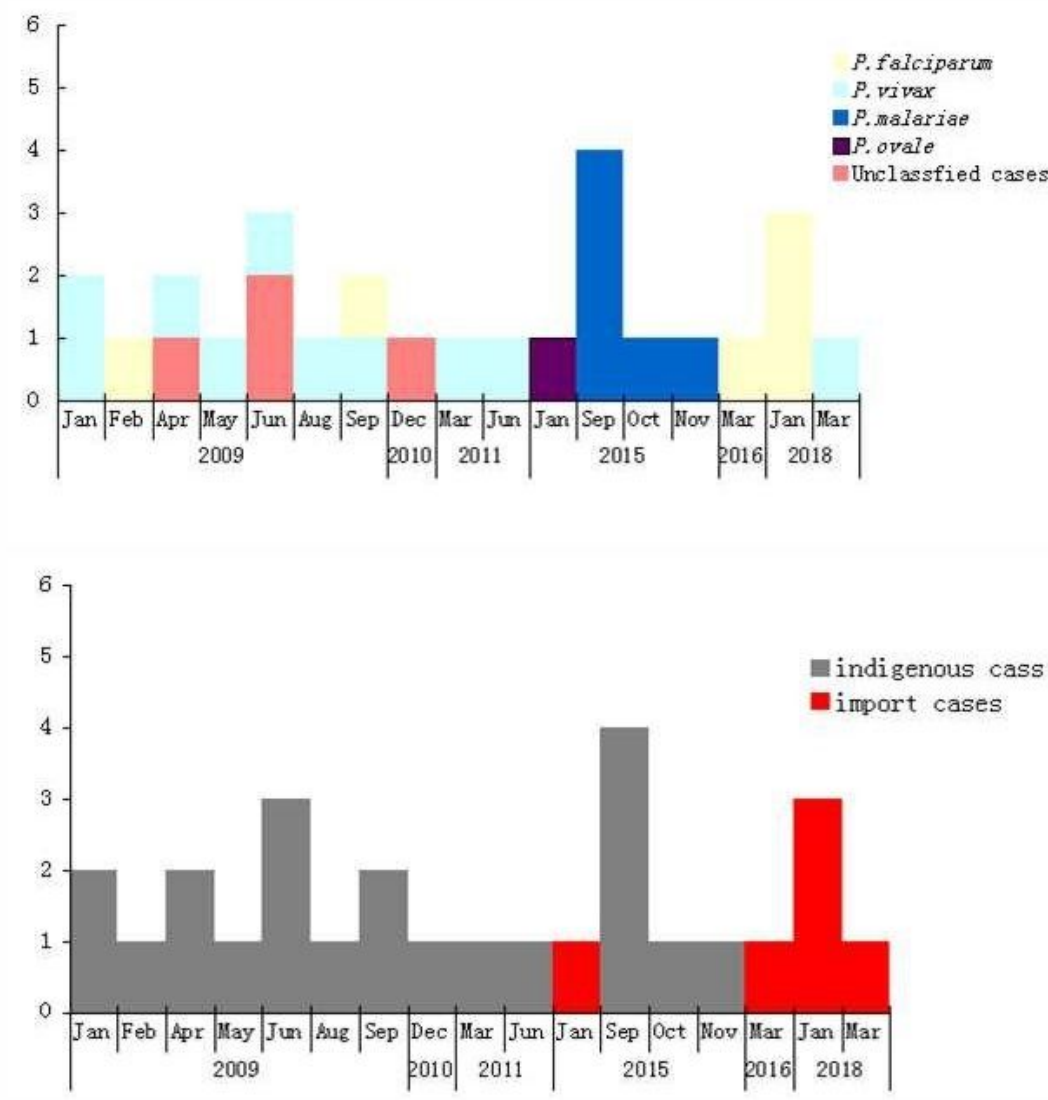

Figure 1

History of malaria surveillance in Sanya from 2009 to 2018, including 6 cases of P. falciparum malaria, 10 cases of P. vivax malaria, 6 cases of P. malariae malaria, 1 case of P. ovale malaria, and 4 cases of unclassified malaria. During the malaria elimination phase from 2011 to 2018 , a total of 14 cases of malaria were reported in Sanya city, including 8 indigenous cases and 6 imported cases. 


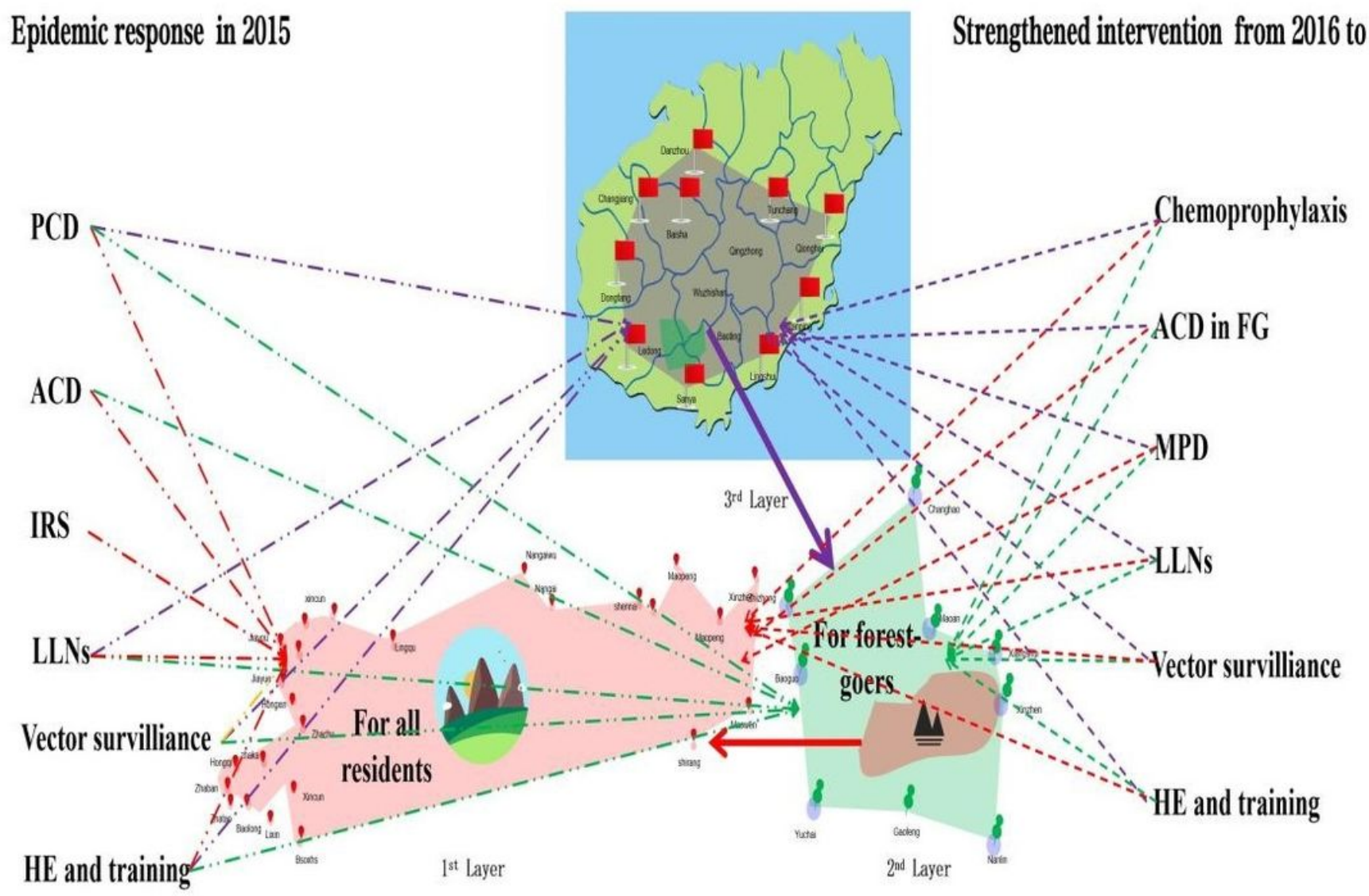

Three-layer strategy (TLS)

Figure 2

TLS which integrated malaria JPCS and the 1-3-7 approach applied to the disposal of the outbreak in Sanya in 2015, and its was also applied to strengthen epidemic measures to prevent emergence by residual foci from 2016 to 2018. 


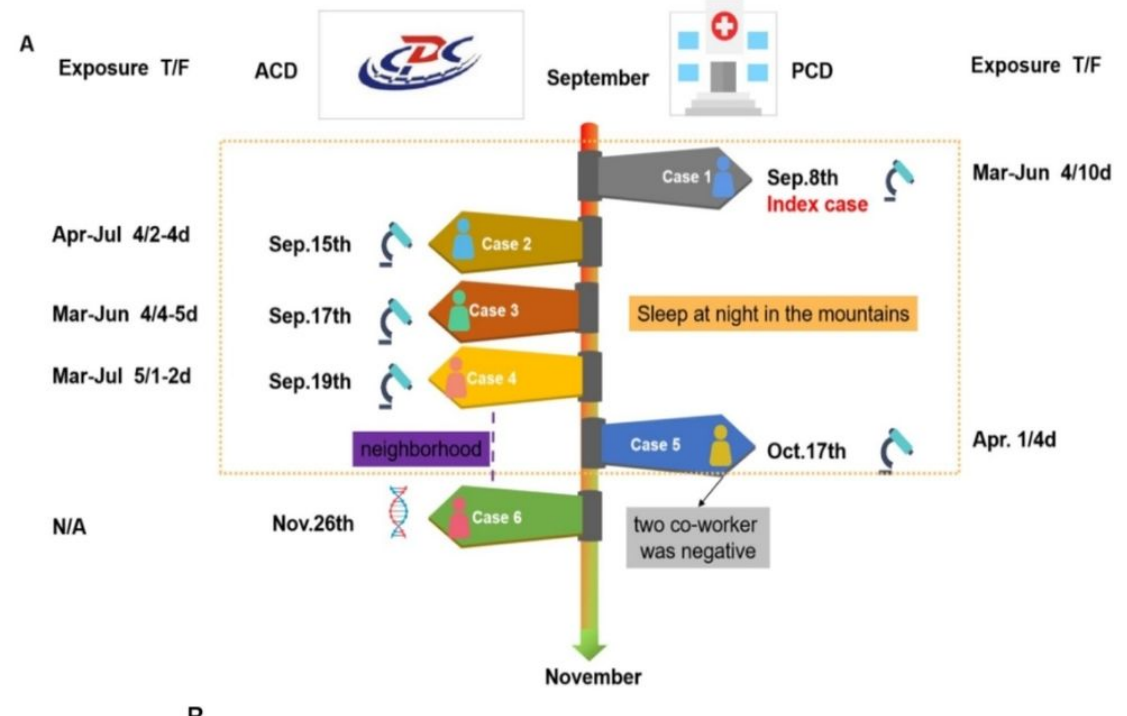

B

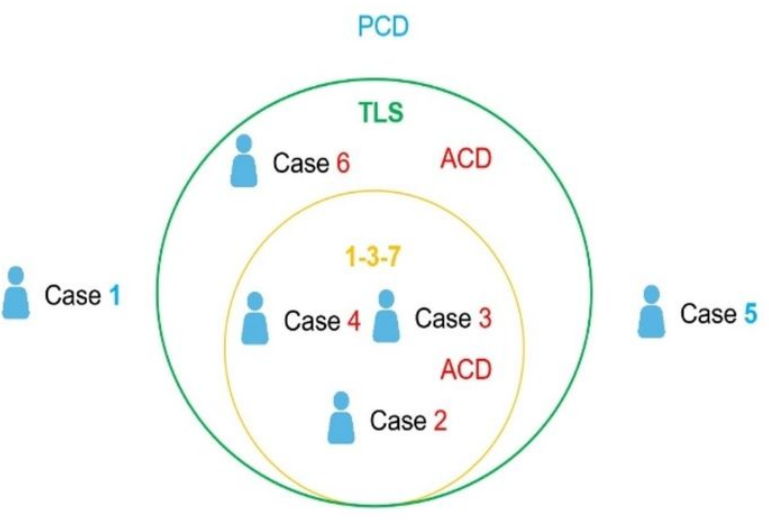

\section{Figure 3}

A. The information of schedule on cases' exposure time and frequency, the way of detection, diagnosis and epidemiological characteristics in this outbreak. B.Comparison of 1-3-7 approach and TLS in case detection ability 